\title{
Predictors of live birth and pregnancy success after in vitro fertilization in infertile women aged 40 and
} over

\author{
Hye Ok Kim ${ }^{1}$, Nayoung Sung ${ }^{2}$, In Ok Song ${ }^{1}$
}

'Division of Reproductive Endocrinology and Infertility, Department of Obstetrics and Gynecology, Cheil General Hospital and Women's Healthcare Center, Dankook University College of Medicine, Seoul; ${ }^{2}$ Department of Obstetrics and Gynecology, Pusan National University Yangsan Hospital, Pusan National University School of Medicine, Yangsan, Korea

Objective: The aim of this study was to evaluate pregnancy outcomes and the live birth rate at 1-year age increments in women aged $\geq 40$ years undergoing fresh non-donor in vitro fertilization (IVF) and embryo transfer (ET), and to identify predictors of success in these patients.

Methods: This retrospective study was performed among women $\geq 40$ years of age between 2004 and 2011. Of the 2,362 cycles that were conducted, ET was performed in 1,532 (73.1\%).

Results: The clinical pregnancy rate and live birth rate in women $\geq 40$ years significantly decreased with each year of increased age $(p<0.001)$. Maternal age (odds ratio [OR], $0.644 ; 95 \%$ confidence interval [Cl], $0.540-0.769 ; p<0.001$ ), basal follicle-stimulating hormone (FSH) levels (OR, $0.950 ; 95 \% \mathrm{Cl}, 0.903-0.999 ; p=0.047)$, the number of high-quality embryos $(\mathrm{OR}, 1.258 ; 95 \% \mathrm{Cl}, 1.005-1.575 ; p=0.045)$, and the number of transferred embryos $(\mathrm{OR}, 1.291 ; 95 \% \mathrm{Cl}, 1.064-1.566 ; p=0.009)$ were significant predictors of live birth. A statistically significant increase in live birth rates was seen when $\geq 3$ embryos were transferred in patients 40 to 41 years of age, whereas poor pregnancy outcomes were seen in patients $\geq 43$ years of age, regardless of the number of transferred embryos. Moreover, the cumulative live birth rate increased in patients 40 to 42 years of age with repeated IVF cycles, but the follicle-stimulating hormone in those $\geq 43$ years of age rarely showed an increase.

Conclusion: IVF-ET has acceptable outcomes in those $<43$ years of age when a patient's own oocytes are used. Maternal age, basal FSH levels, and the number of high-quality embryos and transferred embryos are useful predictors of live birth.

Keywords: Age; Female; Fertilization in vitro; Humans; Infertility; Live birth; Pregnancy

\section{Introduction}

In recent times, pregnancy age has steadily increased, as women have become more socially active and marry at a later age. In Korea,

Received: Nov 17, 2016 · Revised: Feb 15, 2017 · Accepted: Mar 12, 2017 Corresponding author: In Ok Song

Division of Reproductive Endocrinology and Infertility, Department of Obstetrics and Gynecology, Cheil General Hospital and Women's Healthcare Center, Dankook University College of Medicine, 17 Seoae-ro 1-gil, Jung-gu, Seoul 04619 , Korea

Tel: +82-2-2000-7549 Fax:+82-2-2000-7790 E-mail: inok222@naver.com

*This work was orally presented at the fifth Korea-Japan Assisted Reproductive Technology Conference in Busan, Korea in August 24, 2013.

This is an Open Access article distributed under the terms of the Creative Commons Attribution Non-Commercial License (http://creativecommons.org/licenses/by-nc/4.0/) which permits unrestricted non-commercial use, distribution, and reproduction in any medium, provided the original work is properly cited. the number of women giving birth in the age range of 40 to 44 years has nearly doubled from 1997 to 2010 [1]. A similar pattern has emerged worldwide in assisted reproductive technology (ART) programs. In the United States, $19 \%$ of all infertile women who undergo ART procedures are $\geq 40$ years of age [2]. In Europe, the percent of infertile women aged 40 or over undergoing ART was reported to be $15.4 \%$, with a value of $13 \%$ for in vitro fertilization (IVF) and intracytoplasmic sperm injection cycles [3]. In Korea, the percent of women $\geq 40$ years of age who used fresh IVF increased sharply from $11.9 \%$ in 2009 to $19.7 \%$ in 2010 [4].

Fecundity decreases significantly as a woman ages. According to a study by Tietze [5], approximately $10 \%$ of women cannot achieve natural fertility at ages $\leq 34$ years, whereas $87 \%$ cannot achieve natural fertility at ages $\geq 45$ years. While ART may overcome the age-re- 
lated decline in fertility among women of advanced age to some extent, ART success rates are also markedly lower among infertile women of advanced age [6-9]. This tendency has been reported to be associated with reduced oocyte numbers and quality with increased age, along with a higher aneuploidy rate. The increased aneuploidy rate has been reported to be closely associated with an increased rate of spontaneous abortion [10-12]. When ART with donated oocytes is used to overcome low pregnancy rates, higher pregnancy rates can be achieved in aged women [13-15]. However, in Korea and some other countries, most aged infertile women prefer IVFembryo transfer (ET) procedures with their own oocytes over receiving oocyte donation due to issues involving their pedigree, as well as for ethical and social reasons. Therefore, when infertile women undergo IVF-ET using their own oocytes, physicians must provide databased counseling on the pregnancy success rate.

The purpose of the present study was to evaluate pregnancy outcomes in women $\geq 40$ years undergoing IVF-ET using their own oocytes, analyzing how outcomes changed with each additional year of age, and to identify useful predictors of increased success rates in these patients.

\section{Methods}

This retrospective study drew from the institutional IVF database to obtain information on IVF-ET cycles, regardless of the cause of infertility, performed in women over age 40 at the IVF center of Cheil General Hospital and Women's Healthcare Center between January 1, 2004 and December 31, 2011. Only IVF cycles resulting from fresh ET with the patient's own oocytes were analyzed, excluding donor oocytes and frozen embryo cycles. Patients' age at the beginning of the cycle was categorized into 1-year age increments, and data were compared among these groups. All women underwent measurements of basal follicle-stimulating hormone (FSH), basal luteinizing hormone, and estradiol $\left(E_{2}\right)$ levels on menstrual cycle day 2 to 3.

This study was approved by the institutional research ethics committee (No. CGH-IRB-2015-10). Informed consent could not be obtained, as this study involved a retrospective medical record analysis over the course of 10 years.

Controlled ovarian hyperstimulation $(\mathrm{COH})$ was achieved either by short or long protocols using the gonadotropin-releasing hormone (GnRH) agonist leuprolide (Lucrin; Abbott Korea, Seoul, Korea) or $\mathrm{GnRH}$ antagonist protocols using $0.25 \mathrm{mg}$ of GnRH antagonists (Cetrotide; Merck Serono, Geneva, Switzerland or Orgalutran; ScheringPlough Organon, Oss, the Netherlands). The $\mathrm{COH}$ protocol was chosen based on the patient's age, cause of infertility, basal FSH level, previous ovarian response to gonadotropin, body mass index, and physician preference. The most common starting dosage of recombi- nant FSH and/or human menopausal gonadotropin was 225 to 300 IU/day. Follicle monitoring was started on day 6 by transvaginal ultrasonography. Recombinant human chorionic gonadotropin (hCG) (Ovidrel, Merck Serono) was administered subcutaneously at a 250$\mu \mathrm{g}$ or $500-\mu \mathrm{g}$ dose when a dominant follicle had reached a maximum diameter of $18 \mathrm{~mm}$ or greater. Follicle aspiration was performed approximately 36 hours after hCG administration for oocyte retrieval. Cycle cancellation was based on a combination of factors, including low $E_{2}$ levels and poor follicular response to gonadotropin. IVF was performed using standard procedures. In cases of male factor infertility or a low number of retrieved oocytes, intracytoplasmic sperm injection was performed. Embryos were generally transferred to the uterus on days 2 to 5 after oocyte retrieval. We evaluated the embryo quality using Veeck's classification [16], according to equality of the blastomere, cytoplasmic fragmentation, and cytoplasmic blebbing. We divided embryos into grades I, I-a, II, II-a, and III. We defined high-quality embryos as grade I, grade I-a, and grade II. The number of embryos transferred was determined by the available number of embryos, the number of high-quality embryos, and previously attempted cycles. The luteal phase was supplemented with daily intramuscular injections of $50 \mathrm{mg}$ of progesterone in oil or $400 \mathrm{mg}$ of progesterone administered vaginally. Pregnancy was determined by the serum $\beta$-hCG level 12 days after oocyte retrieval. When the serum $\beta$-hCG level was above $5 \mathrm{mIU} / \mathrm{mL}$, it was repeatedly tested to confirm doubling. Clinical pregnancy was defined as the visualization of an intrauterine gestational sac by transvaginal ultrasonography, together with an appropriate $\beta$-hCG concentration.

Pregnancies were followed to delivery in the database. The live birth rate per transferred cycle was the primary outcome measure. Pregnancy rates and spontaneous abortion rates were also calculated in 1-year age increments.

All statistical analyses were performed using PASW ver. 18.0 (SPSS Inc., Chicago, IL, USA). Continuous variables were presented as mean \pm standard deviation, and categorical variables were expressed as raw numbers or percentages. We compared the number of retrieved oocytes and the number of transferred embryos among women at each age through analysis of variance. Chi-square test, Fisher exact test, and the linear-by-linear association method were used to compare percentages. Multiple logistic regression analysis was used to identify significant predictors. Cumulative live births were calculated with Kaplan-Meier curve analysis according to the number of attempted IVF cycles. The $p$-values $<0.05$ were considered to indicate statistical significance.

\section{Results}

We initiated 2,362 cycles in 1,049 infertile women aged 40 and 


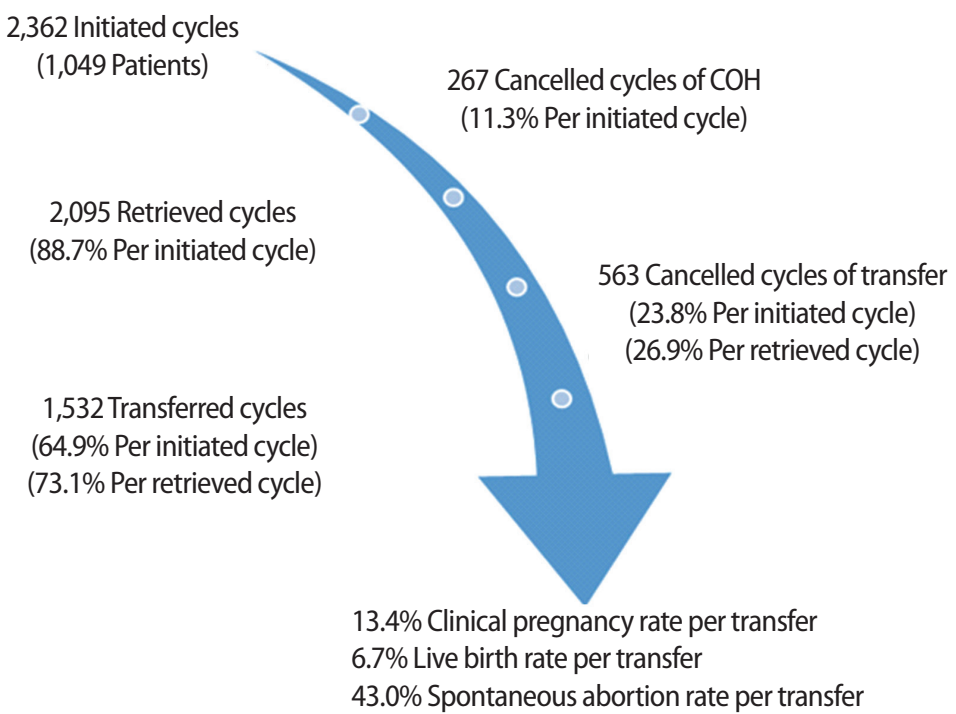

Figure 1. Summary of in vitro fertilization-embryo transfer cycles in this study. $\mathrm{COH}$, controlled ovarian hyperstimulation.

Table 1. IVF-ET parameters in women aged 40 and over

\begin{tabular}{|c|c|c|c|c|c|c|}
\hline Variable & $40 \mathrm{yr}$ & $41 \mathrm{yr}$ & $42 \mathrm{yr}$ & $43 \mathrm{yr}$ & $44 \mathrm{yr}$ & $\geq 45 \mathrm{yr}$ \\
\hline Initiated cycle & 589 & 498 & 385 & 329 & 261 & 300 \\
\hline Basal FSH (mlU/mL) & $12.2 \pm 6.4^{\mathrm{a}, \mathrm{b})}$ & $12.6 \pm 7.8^{\mathrm{c}, \mathrm{d})}$ & $12.5 \pm 6.9^{\mathrm{e}, \mathrm{f})}$ & $12.5 \pm 6.9^{\mathrm{g})}$ & $14.3 \pm 8.6$ & $16.1 \pm 8.8$ \\
\hline Cancelled cycles of $\mathrm{COH}$ ( $\mathrm{n}, \%$ per initiated cycle) & $46(7.8)$ & $50(10)$ & $39(10.6)$ & $35(10.6)$ & $31(11.9)$ & $66(22)$ \\
\hline Retrieved cycle & 543 & 448 & 346 & 294 & 230 & 234 \\
\hline Retrieved oocyte & $6.5 \pm 6.5^{\mathrm{h})}$ & $5.8 \pm 5.7^{(i, j)}$ & $4.9 \pm 4.9^{k, 1)}$ & $4.6 \pm 4.8^{m, n)}$ & $3.2 \pm 3.3$ & $2.6 \pm 3.0$ \\
\hline Fertilization rate (\%) & $58.6 \pm 36.5$ & $56.0 \pm 35.1$ & $62.8 \pm 33.3$ & $54.8 \pm 36.8$ & $55.5 \pm 41.5$ & $59.3 \pm 38.7$ \\
\hline Transferred cycle & 412 & 339 & 280 & 205 & 146 & 150 \\
\hline Transferred embryo & $2.8 \pm 1.2^{0, p)}$ & $2.5 \pm 1.2^{q)}$ & $2.4 \pm 1.2^{r)}$ & $2.5 \pm 1.2^{\mathrm{s})}$ & $2.3 \pm 1.2$ & $2.0 \pm 1.2$ \\
\hline Cancelled cycle at transfer ( $n, \%$ per retrieved cycle) & $131(24.1)$ & $109(24.3)$ & $66(19.1)$ & $89(30.3)$ & $84(36.5)$ & $84(35.9)$ \\
\hline Total cancelled cycle ( $\mathrm{n}, \%$ per initiated cycle) & $177(30.1)$ & $159(31.9)$ & $105(27.3)$ & $124(37.7)$ & $115(44.1)$ & $150(50)$ \\
\hline
\end{tabular}

Values are presented as number or mean \pm standard deviation unless otherwise indicated.

$\mathrm{IVF}$, in vitro fertilization; $\mathrm{ET}$, embryo transfer; $\mathrm{FSH}$, follicle-stimulating hormone; $\mathrm{COH}$, controlled ovarian hyperstimulation.

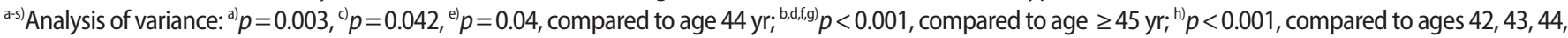
and $\geq 45 \mathrm{yr}^{\text {i) }} p=0.03$, compared to age $43 \mathrm{yr} ;{ }^{j, p)} p<0.001$, compared to ages 44 and $\geq 45 \mathrm{yr}^{,{ }^{k}} p=0.03,{ }^{\mathrm{m})} p=0.041$, compared to age $44 \mathrm{yr}$; ${ }^{(\mathrm{n}, \mathrm{q})} p<0.001$, ${ }^{\text {r) }} p=0.030,{ }^{\text {s) }} p=0.004$ compared to age $\geq 45 \mathrm{yr} ;{ }^{\circ} p=0.001$, compared to age $42 \mathrm{yr}$.

over, which corresponded to $15.7 \%$ of the 15,051 non-donor fresh cycles performed in Cheil General Hospital during that period of time. The indications for IVF-ET in these 1,049 women were unexplained infertility (42\%), ovulatory factor infertility (19\%), tubal factor infertility (18\%), male factor infertility (13\%), endometriosis (5\%), and uterine factor infertility (3\%). In 267 cycles (11.3\%), the oocyte retrieval schedule was cancelled due to poor ovarian response. The mean fertilization rate was $58.0 \% \pm 36.6 \%$. Of the 2,362 cycles that were started, ET was performed in 1,532 (73.1\%). The mean number of retrieved oocytes per cycle was $5.0 \pm 5.4$. The mean number of transferred embryos was $2.5 \pm 1$.2. The overall clinical pregnancy rate per ET was $13.4 \%$. The overall live birth rate per ET was $6.7 \%$, and the spontaneous abortion rate per ET was $43 \%$ (Figure 1).

The data from all 2,362 cycles, subdivided by 1-year age increments at age 40 and over, are presented in Table 1. The cancellation rate increased from $7.8 \%$ at the age of 40 years to $22 \%$ at ages $\geq 45$ years during $\mathrm{COH}$. The total cancellation rate in both $\mathrm{COH}$ and ET was high as $30.1 \%$ at the age of 40 years and $50 \%$ at ages $\geq 45$ years. The clinical pregnancy rate decreased from $22.3 \%$ at the age of 40 years to $2.7 \%$ at ages $\geq 45$ years. The live birth rate decreased from $12.9 \%$ at the age of 40 years to $0.7 \%$ at ages $\geq 45$ years. The clinical pregnancy rate and live birth rate in women $\geq 40$ years significantly decreased every year $(p<0.001)$ (Figure 2).

When IVF parameters were compared between women $\geq 40$ years of age who had a live birth $(n=102)$ versus those who did not have a live birth $(n=1,430)$, the live birth group had a significantly lower maternal age ( $40.9 \pm 1.2$ years vs. $42.0 \pm 1.9$ years, $p<0.001)$, lower paternal age ( $41.8 \pm 3.7$ years vs. $42.6 \pm 4.7$ years, $p=0.034)$, and few- 


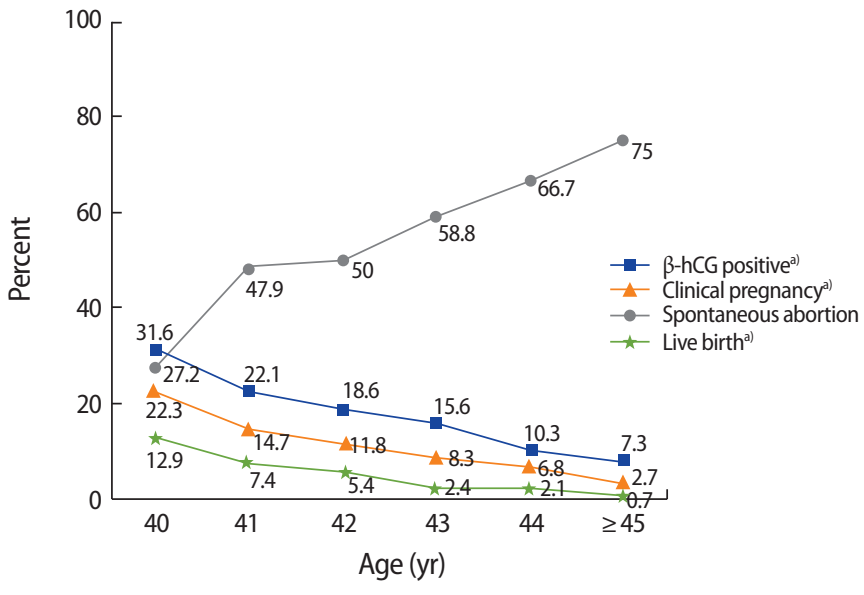

Figure 2. Pregnancy outcomes of in vitro fertilization-embryo transfer cycles in women 40 years of age and older. hCG, human chorionic gonadotropin. ${ }^{\text {a) }} p<0.001$ (linear-by-linear association).

Table 2. Demographic data and IVF parameters in women aged 40 and over according to whether a live birth occurred

\begin{tabular}{lccc}
\hline Variable & Live birth $(+)$ & Live birth $(-)$ & $p$-value \\
\hline Number & 102 & 1,430 & - \\
Maternal age $(\mathrm{yr})$ & $40.9 \pm 1.2$ & $42.0 \pm 1.9$ & $<0.001$ \\
Paternal age $(\mathrm{yr})$ & $41.8 \pm 3.7$ & $42.6 \pm 4.7$ & 0.034 \\
No. of IVF cycles & $2.5 \pm 2.0$ & $3.1 \pm 2.5$ & 0.004 \\
Infertility duration (mo) & $51 \pm 47.3$ & $56.7 \pm 50.4$ & $\mathrm{NS}$ \\
BMI $\left(\mathrm{kg} / \mathrm{m}^{2}\right)$ & $21.6 \pm 2.2$ & $21.7 \pm 2.6$ & $\mathrm{NS}$ \\
Basal FSH $(\mathrm{mIU} / \mathrm{mL})$ & $9.7 \pm 4.4$ & $11.8 \pm 6.2$ & 0.001 \\
$\mathrm{E}_{2}$ on $\mathrm{hCG}$ day $(\mathrm{pg} / \mathrm{mL})$ & $1,628.1 \pm 1,569.2$ & $1,304.1 \pm 1,387.3$ & 0.049 \\
No. of high-quality embryos & $0.57 \pm 0.9$ & $0.36 \pm 0.7$ & 0.028 \\
No. of retrieved oocytes & $8.1 \pm 6.0$ & $5.7 \pm 5.2$ & $<0.001$ \\
No. of transferred embryos & $3.1 \pm 1.1$ & $2.5 \pm 1.2$ & $<0.001$ \\
\hline
\end{tabular}

Values are presented as mean \pm standard deviation.

IVF, in vitro fertilization; NS, not significant; BMI, body mass index; $\mathrm{FSH}$, follicle-stimulating hormone; $\mathrm{E}_{2}$, estradiol; $\mathrm{hCG}$, human chorionic gonadotropin. a)The $p$-values were obtained after adjustment for maternal age.

er repeated IVF cycles $(2.5 \pm 2.0$ vs. $3.1 \pm 2.5, p=0.004)$. Moreover, the live birth group showed statistically significantly lower basal FSH levels $(9.7 \pm 4.4 \mathrm{mlU} / \mathrm{mL}$ vs. $11.8 \pm 6.2 \mathrm{mlU} / \mathrm{mL}, p=0.001)$, higher $\mathrm{E}_{2}$ levels on the $\mathrm{hCG}$ day $(1,628.1 \pm 1,569.2 \mathrm{pg} / \mathrm{mL}$ vs. $1,304.1 \pm 1,387.3 \mathrm{pg} /$ $\mathrm{mL}, p=0.049)$, more high-quality embryos $(0.57 \pm 0.9$ vs. $0.36 \pm 0.7$, $p=0.028)$, a higher number of retrieved oocytes ( $8.1 \pm 6.0$ vs. $5.7 \pm$ $5.2, p<0.001)$, and a higher number of transferred embryos (3.1 \pm 1.1 vs. $2.5 \pm 1.2, p<0.001$ ) (Table 2). Based on these findings, multiple logistic regression analysis was performed to determine the predictors of live birth. An analysis of the various factors associated with live birth identified maternal age at cycle initiation (odds ratio [OR], 0.644; $95 \% \mathrm{Cl}, 0.540-0.769 ; p<0.001$ ), the level of basal FSH (OR, 0.950; $95 \%$ $\mathrm{Cl}, 0.903-0.999 ; p=0.047)$, the number of high-quality embryos (OR, $1.258 ; 95 \% \mathrm{Cl}, 1.005-1.575 ; p=0.045)$, and the number of transferred

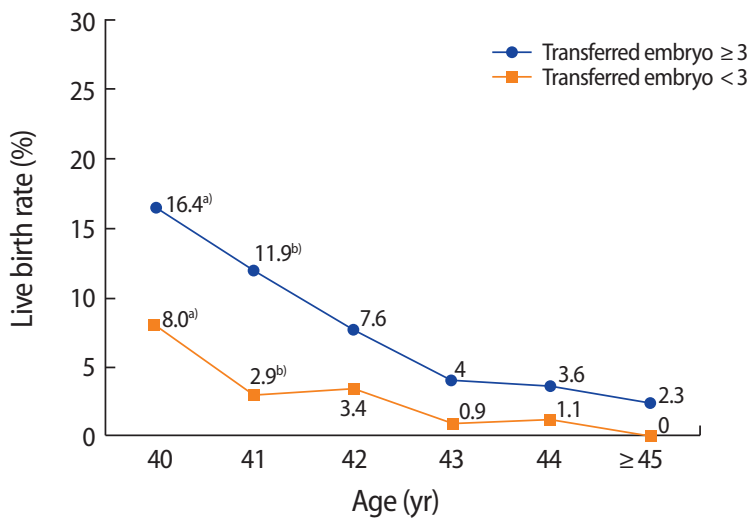

Figure 3. Live birth rate according to the number of transferred embryos. ${ }^{\text {a) }} p=0.017 ;{ }^{\text {b) }} p=0.002$ (Fisher exact test).

Table 3. Predictors of live birth in women aged 40 and over who underwent IVF-ET cycles

\begin{tabular}{lcc}
\hline Predictor & Odds ratio $(95 \% \mathrm{Cl})$ & $p$-value \\
\hline Maternal age & $0.644(0.540-0.769)$ & $<0.001$ \\
Basal follicle-stimulating hormone & $0.950(0.903-0.999)$ & 0.047 \\
Paternal age & $1.015(0.963-1.069)$ & 0.580 \\
Number of IVF cycles & $0.929(0.836-1.033)$ & 0.174 \\
Duration of infertility & $0.997(0.992-1.002)$ & 0.191 \\
Number of retrieved oocytes & $1.026(0.949-1.108)$ & 0.518 \\
Number of high-quality embryos & $1.258(1.005-1.575)$ & 0.045 \\
Number of transferred embryos & $1.291(1.064-1.566)$ & 0.009 \\
\hline
\end{tabular}

IVF, in vitro fertilization; $\mathrm{ET}$, embryo transfer; $\mathrm{Cl}$, confidence interval.

embryos (OR, 1.291; 95\% Cl, 1.064-1.566; $p=0.009)$ as the influencing factors (Table 3). The government financial support program for IVF regulates the number of transferred embryos in infertile women over age 35 years. In this study, a statistically significant increase in the live birth rate was seen when $\geq 3$ embryos were transferred in patients 40 to 41 years of age, whereas poor pregnancy outcomes were seen in patients $\geq 43$ years of age, regardless of the number of embryos transferred (Figure 3). When we compared the IVF parameters in subjects who experienced an abortion $(n=83)$ to those who experienced a live birth $(n=102)$, the only significant difference was maternal age ( $41.6 \pm 1.5$ years vs. $40.9 \pm 1.2$ years, $p=0.001)$. In addition, marginal significance was found for the number of transferred embryos (2.7 \pm 1.2 vs. $3.1 \pm 1.1, p=0.057)$. Other factors, including paternal age, primary infertility history, number of repeated IVF cycles, body mass index, infertility duration, IVF protocol, the total gonadotropin dose used, stimulation days, $\mathrm{E}_{2}$ concentration on the $\mathrm{hCG}$ day, and the rate of high-quality embryos, showed no differences between these two groups.

An analysis of the cumulative live birth rate according to the number of IVF cycles attempted for each age showed a gradually increasing trend in the cumulative live birth rate for up to 11 cycles (40\%) in 


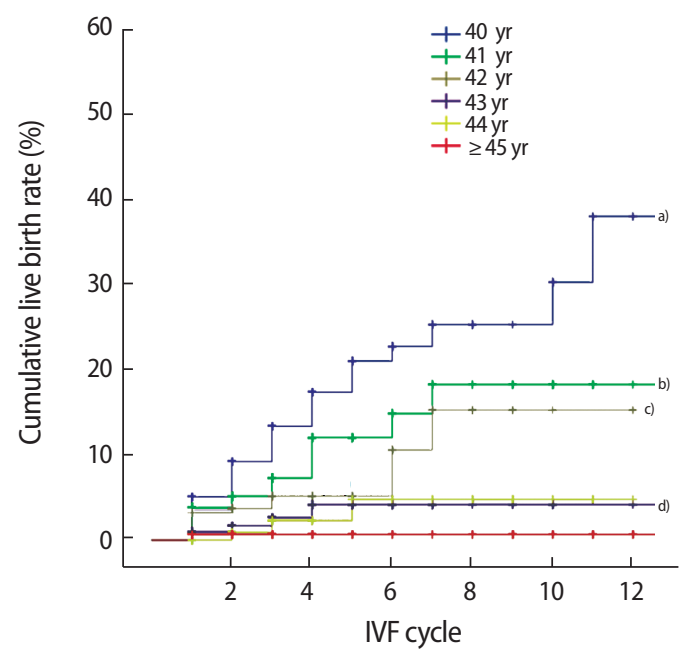

Figure 4. Cumulative live birth rate (CLR) in patients who underwent repeated in vitro fertilization (IVF)-embryo transfer attempts. ${ }^{\text {a) }} \mathrm{A}$ CLR of $40 \%$ in 11 IVF cycles at age $40 \mathrm{yr} ;{ }^{\text {b) }} 19 \%$ in 7 IVF cycles at age $41 \mathrm{yr}$; c) $17 \%$ in 7 IVF cycles at age $42 \mathrm{yr}$, ${ }^{\text {d) }} 3 \%$ in 4 IVF cycles at ages 43 and 44 $\operatorname{yr}(p<0.001)$.

those aged 40 years, up to 7 cycles (19\%) in those aged 41 to 42 years, and up to 4 cycles (3\%) in those aged 43 and 44 years $(p<$ 0.001 ). However, in those $\geq 45$ years of age, repeated attempts did not result in an increased cumulative live birth rate (Figure 4).

\section{Discussion}

When infertile women $\geq 40$ years of age undergo IVF-ET using their own oocytes, pregnancy rates and live birth rates decline in comparison to younger women or when an oocyte donation cycle is performed. Despite these poor reproductive outcomes, IVF procedures among women $\geq 40$ years of age who use their own oocytes rather than donor oocytes continue to increase in some countries, including Korea, for cultural and social reasons. Recently, efforts have been made to promote oocyte freezing for the purpose of fertility preservation in unmarried women of advanced age, but there are still many limitations with respect to the success rate of this technique $[17,18]$.

The age of the women is most important factor in determining pregnancy success rates after IVF-ET. Especially in women $\geq 40$ years of age, a single additional year in age can correspond to a drastic decline in fertility. Klipstein et al. [19] and Serour et al. [20] reported a clinical pregnancy rate of $\leq 10 \%$ and a live birth rate of $\leq 5 \%$ among infertile women $\geq 43$ years of age, indicating that their outcomes declined drastically in comparison with subjects who were 41 and 42 years old. Women $\geq 43$ years of age in the present study showed a similar pattern of a drastic decline in reproductive outcomes in comparison to women 40 to 42 years old, which agrees with the results reported by Klipstein et al. and Serour et al.
Miller et al. [21] demonstrated that unexplained infertility was twice as common in older women than in younger women. Klipstein et al. [19] and Cetinkaya et al. [22] found the most prevalent cause of infertility to be unexplained infertility, accounting for $20 \%$ to $41 \%$ of patients. In the present study, unexplained infertility similarly accounted for $42 \%$ of patients undergoing IVF, in accordance with the results of those studies. It is believed that the reason for such a high incidence of unexplained infertility among women $\geq 40$ years of age may be that IVF procedures are expedited with increased age out of concerns regarding a decline in oocyte numbers and quality, or due to diminished ovarian reserve.

Clinical pregnancy rates continue to improve due to advances in IVF techniques. Nonetheless, the live birth rate among women $\geq 43$ years of age remains low (2\% to $3 \%)[9,19]$. The present study also found low live birth rates of $2.4 \%, 2.1 \%$, and $0.7 \%$ in subjects who were 43,44 , and $\geq 45$ years old, respectively, with women $\geq 45$ years of age showing a spontaneous abortion rate of almost $75 \%$. Most of these losses can be expected to be chromosomal in origin [9]. Although some physicians perform preimplantation genetic screening to overcome these low live birth rates and chromosomal abnormalities, there are many limitations in applying preimplantation genetic screening in patients $\geq 43$ years of age who are poor responders [12]. The present study investigated infertile women $\geq 40$ years of age from a single center for the purpose of identifying the predictors of acceptable pregnancy success at 1-year age increments. Klipstein et al. [19] analyzed the factors that influenced pregnancy outcomes in 2,705 cycles in patients $\geq 40$ years of age and reported that higher live birth rates were seen in patients with a greater number of transferable embryos and a greater number of cryopreserved embryos. In the present study, live birth rates were also higher in cases with $\geq 3$ transferred embryos than in cases with $<3$ transferred embryos, similarly to the results of Klipstein et al. Therefore, these results could be useful for predicting patient outcomes prior to IVF-ET procedures in women $\geq 40$ years of age.

Unlike previous studies, the present study analyzed cumulative live birth rates according to the number of IVF cycles when IVF-ET procedures were attempted repeatedly in each age group among patients $\geq 40$ years of age. The cumulative live birth rates showed a gradually increasing trend up to 11,7 , and 3 cycles in those aged 40, 41-42, and 43-44 years, respectively, while repeated attempts did not increase the live birth rate among those $\geq 45$ years of age. Malizia et al. [23] reported that among patients who were 40 years of age or older, the cumulative live birth rate after 6 cycles was $42 \%$ with an optimistic analysis and $23 \%$ with a conservative analysis. Cetinkaya et al. [22] reported that in older patients ( $>43$ years) the cumulative live birth rate went from $1.1 \%$ in the first cycle to $9.1 \%$ after 3 cycles. They recommended that oocyte donation should be considered thereafter in 
patients $\geq 43$ years of age, since IVF outcomes improved over the course of three attempts. However, women in Korea often wish to continue IVF using their own oocytes after numerous failed IVF cycles, even if doing so is associated with poor outcomes, instead of switching to an oocyte donor program, because of the lack of oocyte banks and social considerations that place a high value on pedigree. Homburg et al. [24] indicated that an acceptable clinical pregnancy rate (11.2\%) can be achieved by attempting $\geq 12$ cycles, and also reported the occurrence of a successful pregnancy after $\geq 20$ cycles. Although that study did not analyze patients $\geq 40$ years of age, Homburg et al. [24] did argue that physicians should not place a specific limit on the number of IVF attempts made by a patient. That study was conducted in Israel, which is believed to have a social context in which pedigree is valued, similarly to Korea. Therefore, it is suggested that our results on cumulative live birth rates in older infertile women will be helpful when counseling patients who have undergone repeated IVF procedures.

In conclusion, this study showed that IVF-ET had acceptable outcomes in women $<43$ years of age using their own oocytes. Maternal age, basal FSH, and the number of high-quality embryos and transferred embryos may be useful predictors of live birth in older infertile women.

\section{Conflict of interest}

No potential conflict of interest relevant to this article was reported.

\section{References}

1. Statistics Korea. Live birth rate by age [Internet]. Daejeon: Statistics Korea; 2011 [cited 2017 Jun 23]. Available from: http://www. index.go.kr/potal/main.

2. Centers for Disease Control and Prevention. 1999 Assisted reproductive technology success rates. Atlanta: Centers for Disease Control and Prevention; 2001.

3. Nyboe Andersen A, Goossens V, Bhattacharya S, Ferraretti AP, Kupka MS, de Mouzon J, et al. Assisted reproductive technology and intrauterine inseminations in Europe, 2005: results generated from European registers by ESHRE: ESHRE. The European IVF Monitoring Programme (EIM), for the European Society of Human Reproduction and Embryology (ESHRE). Hum Reprod 2009;24:1267-87.

4. Lee GH, Song HJ, Lee KS, Choi YM. Current status of assisted reproductive technology in Korea, 2010. Clin Exp Reprod Med 2015;42:8-13.

5. Tietze C. Reproductive span and rate of reproduction among Hutterite women. Fertil Steril 1957;8:89-97.

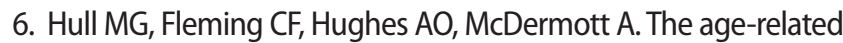
decline in female fecundity: a quantitative controlled study of implanting capacity and survival of individual embryos after in vitro fertilization. Fertil Steril 1996;65:783-90.

7. Lass A, Croucher C, Duffy S, Dawson K, Margara R, Winston RM. One thousand initiated cycles of in vitro fertilization in women > or $=40$ years of age. Fertil Steril 1998;70:1030-4.

8. Baird DT, Collins J, Egozcue J, Evers LH, Gianaroli L, Leridon H, et al. Fertility and ageing. Hum Reprod Update 2005;11:261-76.

9. Spandorfer SD, Bendikson K, Dragisic K, Schattman G, Davis OK, Rosenwaks Z. Outcome of in vitro fertilization in women 45 years and older who use autologous oocytes. Fertil Steril 2007; 87:74-6.

10. Gianaroli L, Magli MC, Ferraretti AP, Munne S. Preimplantation diagnosis for aneuploidies in patients undergoing in vitro fertilization with a poor prognosis: identification of the categories for which it should be proposed. Fertil Steril 1999;72:837-44.

11. Smith KE, Buyalos RP. The profound impact of patient age on pregnancy outcome after early detection of fetal cardiac activity. Fertil Steril 1996;65:35-40.

12. Munne S, Cohen J, Sable D. Preimplantation genetic diagnosis for advanced maternal age and other indications. Fertil Steril 2002;78:234-6.

13. Sauer MV, Paulson RJ, Lobo RA. Pregnancy after age 50: application of oocyte donation to women after natural menopause. Lancet 1993;341:321-3.

14. Paulson RJ, Boostanfar R, Saadat P, Mor E, Tourgeman DE, Slater CC, et al. Pregnancy in the sixth decade of life: obstetric outcomes in women of advanced reproductive age. JAMA 2002; 288:2320-3.

15. Yeh JS, Steward RG, Dude AM, Shah AA, Goldfarb JM, Muasher SJ. Pregnancy outcomes decline in recipients over age 44: an analysis of 27,959 fresh donor oocyte in vitro fertilization cycles from the Society for Assisted Reproductive Technology. Fertil Steril 2014;101:1331-6.

16. Veeck LL. The morphological assessment of human oocytes and early concepti. In: Keel BA, Webster BW, editors. Handbook of the laboratory diagnosis and treatment of infertility. Boca Raton: CRC Press; 1990. p. 353-70.

17. Argyle CE, Harper JC, Davies MC. Oocyte cryopreservation: where are we now? Hum Reprod Update 2016;22:440-9.

18. Tsafrir A, Haimov-Kochman R, Margalioth EJ, Eldar-Geva T, Gal M, Bdolah Y, et al. Ovarian stimulation for oocyte cryopreservation for prevention of age-related fertility loss: one in five is a low responder. Gynecol Endocrinol 2015;31:779-82.

19. Klipstein S, Regan M, Ryley DA, Goldman MB, Alper MM, Reindollar RH. One last chance for pregnancy: a review of 2,705 in vi- 
tro fertilization cycles initiated in women age 40 years and above. Fertil Steril 2005;84:435-45.

20. Serour G, Mansour R, Serour A, Aboulghar M, Amin Y, Kamal O, et al. Analysis of 2,386 consecutive cycles of in vitro fertilization or intracytoplasmic sperm injection using autologous oocytes in women aged 40 years and above. Fertil Steril 2010;94:1707-12.

21. Miller JH, Weinberg RK, Canino NL, Klein NA, Soules MR. The pattern of infertility diagnoses in women of advanced reproductive age. Am J Obstet Gynecol 1999;181:952-7.
22. Cetinkaya MB, Siano L, Benadiva C, Sakkas D, Patrizio P. Reproductive outcome of women 43 years and beyond undergoing ART treatment with their own oocytes in two Connecticut university programs. J Assist Reprod Genet 2013;30:673-8.

23. Malizia BA, Hacker MR, Penzias AS. Cumulative live-birth rates after in vitro fertilization. N Engl J Med 2009;360:236-43.

24. Homburg R, Meltcer S, Rabinson J, ScharfS, Anteby EY, Orvieto R. Is there a limit for the number of in vitro fertilization cycles for an individual patient? Fertil Steril 2009;91(4 Suppl):1329-31. 